\title{
A Novel Approach to Mitigating Neointimal Hyperplasia Using Kohn-Sham DFT and Free-Radical Polymerization
}

\author{
Anish C. Pothireddy, Samyak R. Thapa, Vishnu Lakshmanan \\ Governor's School at George Mason University, 10900 University Blvd, Manassas, VA 20110, U.S.A.; anish.c.pothireddy@gmail.com
}

\begin{abstract}
Pancreatic adenocarcinoma is one of the most common gastrointestinal cancers and is often associated with a poor prognosis. To improve patient outcomes, an en bloc resection of the portal and mesenteric vein is suggested. However, it has a high failure rate of $81 \%$ caused by graft stenosis and occlusion from neointimal hyperplasia, which is the proliferation of vascular smooth muscle cells within the vascular wall. Polytetrafluoroethylene is the material used in constructing venous grafts, but we propose a replacement polymer coated with poly ( $\mathrm{N}$-isopropylacrylamide) nanoparticles for steady drug release to limit proliferation. Kohn-Sham Density Functional Theory was used to compare the bulk modulus of two polymers, Polytetrafluoroethylene and Polyethylene Terephthalate, and free-radical polymerization with swelling tests were used to determine the optimal chemical concentrations for nanoparticle synthesis. We found that polyethylene terephthalate had a higher bulk modulus than Polytetrafluoroethylene by 16.6 gigapascals, making it more viable as graft material, and that $75 \% \mathrm{~N}$-isopropylacrylamide, $25 \%$ $\mathrm{N}, \mathrm{N}-\mathrm{Methylenebisacrylamide,} \mathrm{and} 42.5 \%$ Poly-vinyl alcohol were the optimal concentrations of chemicals for nanoparticle synthesis. These particles had an average 48-hour swelling ratio of 38\%. From these results, a new graft was proposed, made from PET and coated with PNIPAm nanoparticles with the predetermined concentrations.

KEYWORDS: Neointimal hyperplasia; polymeric nanoparticles; PNIPAm; drug delivery; density functional theory.
\end{abstract}

\section{- Introduction}

According to the American Cancer Society, 1,762,450 new cancer cases were diagnosed in 2019. Out of those diagnosed with cancer that year, 606,880 patients died. ${ }^{1}$ Cancer has quickly become one of the most devastating diseases in humankind. The uncontrolled growth of malignant cells, invading various organs, has proven a formidable opponent for medical professions. Pancreatic adenocarcinoma or pancreatic cancer, though not the leading cause of deaths from cancer, is known for its incredibly low survival rate due to the time it takes to diagnose the disease. Pancreatic cancer is difficult to catch in its early stages due to the location of the tumor and the asymptomatic patient response until the final stages of the cancer. If the cancer is caught in time with possible hope for remission, doctors perform a pancreaticoduodenectomy, a procedure where the cancerous portion of the pancreas, parts of the small intestine, gallbladder, and in severe cases the portal vein or superior mesenteric vein is resected. A collaborative study between Drs. Katharina Marsoner, Rainer Langeder, Dora Csengeri, Hans Jörg Mishchinger, and Peter Kornprat at the Medical University of Graz and Dr. Gottfried Sodeck at the Medical University of Vienna worked to determine the effects of portal vein resection on patient survival and its viability in becoming the standard in pancreatic cancer treatment. ${ }^{2}$ They assessed survival rates and postoperative complication rates to conclude that patients who underwent portal vein resection did not face any lower survival rates or any higher postoperative complication rates. The researchers, however, were able to conclude that from an oncological point of view, portal vein resection "is the only chance" for patients with lo- cally advanced pancreatic cancer to achieve similar survival rates comparable to early stage cancer patients. ${ }^{2}$ With portal vein resection quickly becoming a new standard, a new field of study in venous reconstruction has developed, resulting in new and innovative methods for combating common complications. This study endeavored to address the optimal method of mitigating a common complication, neointimal hyperplasia, post venous reconstruction. The researchers gathered data in a quasi-experimental design setting over a nine-month period at a mid-Atlantic research university.

To determine the most common complication arising from venous reconstruction and the best method of developing a preventative measure, it is necessary to perform a review of existing literature. First, studies conducted in 2016 were analyzed to determine current forms of venous reconstruction, success rates, and sources of complication. Second, density functional theory and its applicability to the methods of this study was explored. Next, free-radical polymerization with $\mathrm{N}$-isopropylacrylamide (NIPAm) was analyzed and was determined to be the most relevant method of synthesizing polymers. Lastly, NIPAm nanoparticles were researched to determine their applicability as a form of targeted drug delivery.

\section{Literature Review}

\section{Current forms of Venous Reconstruction}

The reconstruction of the Portal and Superior Mesenteric Vein is required when a patient undergoes surgery for pancreatic cancer. Currently, after the surgery for removing the tumor is performed, the primary surgeon inserts a graft that 
is made from polytetrafluoroethylene (PTFE). Polytetrafluoroethylene is primarily used because of its believed durability and resistance to bacterial growth compared to other materials. In Dr. Farzard Alemi et al.'s research, he discusses how the surgery should be performed in the area of interest so that the placement and patency of the graft would be optimal. In performing the surgery, he laid out 5 different zones: hepatic hilum, hepatoduodenal ligament, portal vein/splenic vein confluence, infra-confluence, and the splenic vein. These five zones are where the veins of interest are located and where the graft will be inserted during the operation. They also described that the surgery should be done by adding the vascular clamps first, and then removing the gland so that the graft can be placed in afterwards. Backup procedures are also included in the case of an unexpected event. The researchers also detailed the procedure for the attachment and reconstruction of the graft so that it is placed in the right area with the necessary fit so that it is not loose and does not constrict any functions inside the body. Throughout these five zones however, these grafts have almost an 80 percent failure rate. The research performed by Dr. Suh Min Kim et al. ${ }^{4}$ explored the reconstruction of the portal vein and the superior mesenteric vein. Their objective was to see how venous reconstruction could work if it was encountered during surgery for pancreatic cancer. The researchers analyzed the effects of reconstruction and performance over time for two different veins. When these grafts were placed in the human body, human error was a secondary reason for the high failure rate with graft occlusion being the primary cause. ${ }^{5}$ Dr. Dyre Kleive et al. attempted to determine the feasibility and surgical outcomes of using venous allografts in the reconstruction of the portal vein and the superior mesenteric vein in patients who underwent pancreatic resection. ${ }^{5}$ Through this experiment, it was determined that many patients died after surgery and before discharging due, in part, to installation error but primarily graft stenosis and restriction of the luminal diameter. Dr. Kleive et al.'s research demonstrated the current method of applying allografts for venous reconstruction and how effective the graft was in terms of patency, presenting information on the methods that are currently used in reconstructing the Portal and Superior Mesenteric Vein. Additionally, another study found that the grafts that are created and placed inside patients may cause other issues such as cardiac arrest, thrombosis, and multi-organ failure within ninety days, and in this study, the mortality rate of those tested was $4.4 \% .^{6}$ This can ultimately be attributed to the inability of grafts to combat graft occlusion and stenosis, demonstrating an increasing necessity for an adequate alternative.

\section{Density Functional Theory and Quantum ESPRESSO}

Density functional theory can be used to determine the mechanical properties of chemicals by utilizing Kohn-Sham Density Functional Theory and solving an approximation to the Schrödinger equation. Quantum ESPRESSO uses "local-density approximation to quantum mechanical exchange correlation effects" to solve the approximation. ${ }^{7}$ Self-consistent field (SCF) calculations are run in a loop until convergence with the total energy being the desired data point for analysis. A collaborative study in 2018 between Stanford University and the National Institute of Standards and Technology describes how elastic tensor is a critical property in determining mechanical properties such as bulk modulus and elastic deformation. ${ }^{8}$ The researchers describe how conventional methods such as ultrasonic measurement and nanoindentation are limited by experimental data but density functional theory (DFT) can fill in the gap and is applicable to a wider range of materials. In their methodology, there are consistent references to the Material Project (MP) which provides a large database of compounds and their respective bond and chemical properties. Crystalline structures and related information necessary for DFT computations can be found using the MP. Furthermore, in their study, the researchers categorized thousands of materials and their mechanical properties by utilizing DFT calculations, supporting its application in the context of this study. Pure, orbital free, DFT is based largely on computing the energy of interacting electrons as a function of density. This approach has proven correct in theory but when placed into practice, the lack of accurate approximations for the kinetic energy functional, severely limits the applicability of pure DFT computations. Kohn-Sham Density Functional Theory attempts to solve this problem by working with a system of non-interacting electrons. While the density of a non-interacting system is different from that of an interacting system, Kohn-Sham DFT constructs a fictional non-interacting system which has the same density as that of an interacting system. ${ }^{9}$ This lowers the computational cost while maintaining the accuracy of the simulation and calculations.

\section{NIPAm and Polymerization}

A fundamental aspect of this study lies in the crosslinking of NIPAm with N,N'-Methylenebisacrylamide. These two materials were chosen specifically because they are substances which are biologically compatible and have the necessary attributes to function well within the body. Additionally, they are often cross-linked because they strengthen the positive attributes of one another, most important being biological compatibility. According to research conducted by Dr. Sonia Lanzalaco and Dr. Elaine Armelin, ${ }^{10}$ NIPAm specifically has many practical applications, including "controlled delivery of active molecules, in self-healing materials, tissue engineering, regenerative medicine, or in the smart encapsulation of cells." Additionally, one important characteristic of NIPAm is its biocompatibility. The researchers listed different methods of achieving biocompatibility, among which include using dextrin, Bis, and Potassium Persulfate. Additionally, the article lists several useful methods of crosslinking NIPAm, including free radical polymerization, atom transfer radical polymerization, and copolymerization. The research suggests that NIPAm linked with acrylic acid produces a biocompatible biomaterial. The step-by-step analysis of NIPAm's behavior will prove useful in our research, as NIPAm will be the main polymer used for our synthesized particles. Furthermore, the potential applications of NIPAm copolymerization, which include cell adhesion, regenerative 
medicine, and tissue engineering, support the high biological application of this project.

It has been established that when crosslinked, NIPAm and Bis complement each other's properties. An important step, therefore, is establishing the means to produce such a copolymerization. According to Dr. Xiaobo Hu, Dr. Zhen Tong, and Dr. L. Andrew Lyon, in their paper "Control of Poly(N-isopropylacrylamide) Microgel Network Structure by Precipitation Polymerization near the Lower Critical Solution Temperature," it is possible to achieve a link between NIPAm and Bis using precipitation polymerization. ${ }^{11}$ They state that a "water soluble, free-radical initiator" is needed to start the cross-polymerization. ${ }^{11}$ Regarding our study, since we will be performing emulsion polymerization, we require the utilization of a water-soluble initiator. Potassium Persulfate is one of the most common initiators used in emulsion polymerization and will also be used in this study. Additionally, in this reaction, oxygen is a critical factor to consider because it can slow down the rate of cross-polymerization. To account for this, we will be utilizing Tetramethylethylenediamine (TEMED). TEMED is used as a stabilizer in free-radical polymerization and will be used to speed up the reaction of our polymerization. ${ }^{12}$ TEMED will be the alternative to traditional degassing due to limitations of supply and materials. With these limitations in mind, a critical component of this research project includes how NIPAm works inside the body. A study by Dr. Kenichi Nagase, Dr. Masayuki Yamato, Dr. Hideko Kanazawa, and Dr. Teruo Okano titled "Poly(N-isopropylacrylamide)-based thermo-responsive surfaces provide new types of biomedical applications" describes the application of NIPAm in the human body. ${ }^{13}$ This report outlines various biomedical uses of NIPAm and its biocompatibility. They state that NIPAm "is the most well-known and widely used stimuli-responsive polymer in biomedical fields."13 The biomedical applications include thermo-responsivity and "thermally modulated drug and delivery systems."13 This is significant to our project because it confirms that NIPAm will work within the body in several broad applications, including targeted drug delivery.

\section{NIPAm Based Nanoparticles for Drug Delivery}

A novel application of NIPAm for venous graft would be the implementation of nanoparticles. This could be used to combat neointimal hyperplasia where muscle cells accumulate within the interior of the graft or vein restricting the luminal diameter, making it harder for blood to flow. If cells do build up alongside the wall of the graft, then the NIPAm nanoparticles and their respective thermo-responsive properties could be used to limit cell proliferation, allowing for the blood to flow through. A typical nanoparticle is defined by its shape and size. They are usually between 100 and 2500 nanometers long and are typically circular. To maximize efficiency and guarantee that proliferation will be limited, a spherical shape is mostly used. By using nanoparticles, it is possible to load them with the drug dexamethasone and use them to target the area of cell accumulation. Dexamethasone is a pharmaceutical drug which inhibits the phosphorylation of the $\mathrm{Rb}$ protein. By slowly releasing it and targeting the cells, the chances of graft stenosis and occlusion drastically decrease resulting in optimal blood flow. Another aspect to consider when utilizing nanoparticles is the lower critical solution temperature properties (LCST). The LCST property is the critical temperature below which the components of a mixture are miscible for all the various compositions found. These properties are significant because they are critical to the design and development of certain polymers. The Lower Critical Solution Temperature for NIPAm is thirty-two degrees Celsius (89.6 degrees Fahrenheit) in aqueous solutions. This allows any polymer that contains NIPAm to be fully functional in any form within the human body which is typically within the temperatures in which NIPAm is stable and therefore will not negatively affect the human body. Another aspect that the "LCST properties determine is whether the polymer is thermo-responsive." 14 If the polymer is thermo-responsive, certain aspects may be utilized to further increase nanoparticle efficiency and fluctuate drug release periods.

\section{- Results and Discussion}

After running fifty plane wave self-consistent field calculations per chemical and utilizing Murnaghan's equation to determine the bulk modulus of each chemical based on the total energies from volume, polyethylene terephthalate (PET) demonstrated a significantly higher mechanical composition value compared to polytetrafluoroethylene (PTFE). PET had a computed bulk modulus of $105.9 \mathrm{GPa}$ whereas PTFE had a computed bulk modulus of $89.3 \mathrm{GPa}$ (Table 1). The bulk modulus is a numerical representation of how much pressure is required to alter the volume of a substance. Higher bulk modulus values indicate a sturdier polymer capable of withstanding larger amounts of force, increasing the respective applicability of the polymers in a strenuous context such as within the human body. Apart from the direct comparison of the bulk modulus values for the two chemicals, relevant distinctions arose when analyzing and comparing the two graphs where the data points of volume vs total energy were plotted (Figure 1 and 2). With constant decrements from the starting chemical volume for both PET and PTFE, the calculated total energies began to rise sharply at an exponential growth rate. The smaller volumes had a noticeable correlation to higher energies and as the volume began to reach the natural values, the exponential increase in total energy began to decrease.

Correlation statistics were used to determine the correlation strength between volume and total energy per compound. PET had a correlation value of -0.5049 indicating a negative relationship between the volume data column and total energy data column (Appendix Table 1). The correlation number was predicted to be higher but the apparent inconsistency in total energy fluctuations towards the higher volumes resulted in a lower correlation value. Due to this, PTFE exhibited a higher negative correlation of -0.6072 between the volume data column and the total energy data column (Appendix Table 2). The higher correlation value resulted from more level and consistent values for total energy for the larger volumes as opposed to the inconsistency of respective data points for PET. Furthermore, 
the data set had a $\mathrm{p}$ value of $1.4 \mathrm{E}-133$. This means that our null hypothesis indicating that there was no difference in the bulk modulus values between the two chemicals was incorrect and our alternate hypothesis was supported.

Another important distinction regarding the data was the noticeable fluctuations in CPU computational time for computing the SCF calculations for PTFE when compared to PET. PET convergence was achieved within 14 iterations for most of the trials and total CPU time did not exceed 10 minutes. PTFE, in contrast, fluctuated greatly in CPU time and number of iterations for convergence. For example, the final calculation for PTFE with the largest tested volume took one hour and seventeen minutes to finish computing whereas the previous volume trial took twenty minutes. In addition, the number of iterations for convergence reached 212 iterations, whereas the previous volume calculation reached the convergence threshold within 52 iterations. This may be because the lattice parameters

Table 1. Calculated Bulk Modulus of PTFE and PET with Equilibrium Volume.

\begin{tabular}{|c|c|c|}
\hline Polymer Type & Bulk Modulus & Equilibrium Volume \\
\hline $\begin{array}{c}\text { Polytetrafluoroethylene } \\
\text { (PTFE) }\end{array}$ & $89.3 \mathrm{GPa}$ & $320.02 \mathrm{Ang}^{3}$ \\
\hline $\begin{array}{c}\text { Polyethylene } \\
\text { Terephthalate (PET) }\end{array}$ & $105.9 \mathrm{GPa}$ & $253.12 \mathrm{Ang}^{3}$ \\
\hline
\end{tabular}

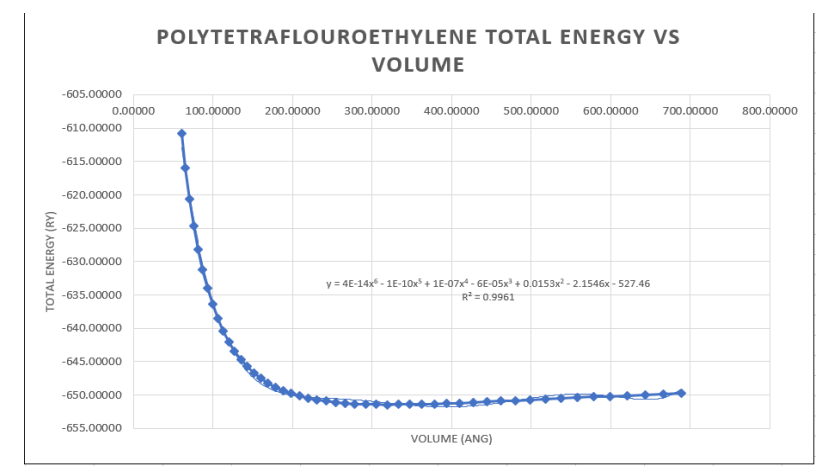

Figure 1 Plotted Data Points for DFT Calculations for PTFE.

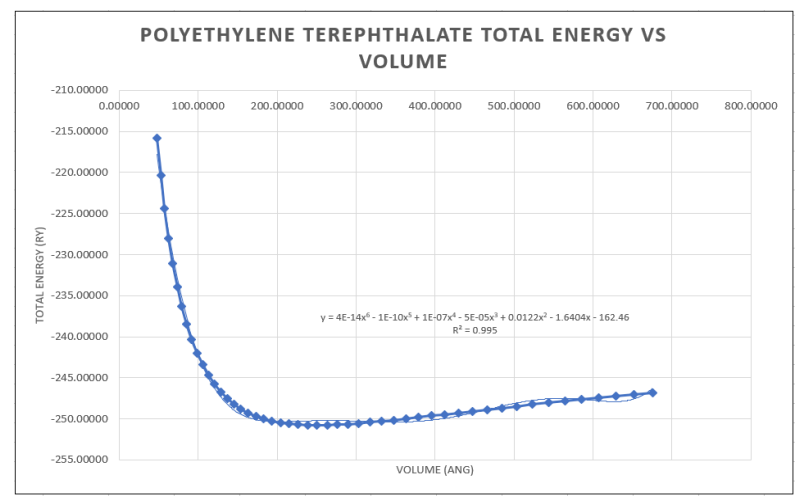

Figure 2 Plotted Data Points for DFT Calculations for PET. reached a certain value where Kohn-Sham Density Functional Theory was unable to create a non-interacting system of particles with the same density as the original system within the same time frame as the previous trials. Further analysis into computational time and number of iterations revealed that a possible way to decrease computational time for large volumes would be to fluctuate the wave function cutoff parameter for larger volumes to achieve convergence quicker, but with the same level of accuracy.

From the polymerization portion of the second phase, the data indicated a critical trend attesting to a previously held hypothesis regarding the point of diminishing returns for the concentrations of Bis and PVA. Bis was initially added as a crosslinker to enhance the desirable properties of NIPAm. The molar concentration of Bis was gradually increased by $5 \%$ to improve the mechanical integrity of the polymer. A Bis concentration of $25 \%$ resulted in a polymer which passed all mechanical tests. To determine if this concentration of Bis was optimal, the molar concentration was increased by $5 \%$ three more times to see if the synthesized polymer would retain its structural integrity, possibly even becoming sturdier. Based on the data, the opposite was observed: as the concentration of Bis exceeded 25\%, the polymer failed to pass the elastic test and passed the shear test only once (Table 2). Compression testing was the only test consistently passed by the polymers. Based on these results, the conclusion was formed that a concentration of $25 \%$ Bis represented the threshold value for Bis concentration before mechanical and structural degradation was noticed in the synthesized polymers. With the optimal concentration of Bis determined, PVA was added incrementally to determine a similar optimal concentration value. Two concentrations were noticed to have passed all three mechanical tests: $40 \%$ and $42.5 \%$ PVA. A similar threshold value was noticed for PVA concentrations: as the concentration passed $42.5 \%$ PVA, the polymer no longer was able to pass all three mechanical tests and failed sheer testing. From mechanical testing, three polymer concentrations were determined to be viable for swelling tests. Overall, a general trend was noticed for the polymers synthesized: The majority of the polymers passed compression testing with only the polymer with $10 \%$ Bis concentration failing. Another critical observation was noticed: the inclusion of PVA resulted in polymers which consistently passed elastic testing, indicating the ability of PVA to create an interpenetrating polymer network which adds elastic properties to synthesized polymers.

Swelling tests were conducted on polymers created from $75 \%$ NIPAm 25\% Bis (Polymer A), 75\% NIPAm 25\% Bis $40 \%$ PVA (Polymer B), and 75\% NIPAm 25\% Bis $42.5 \%$ PVA (Polymer C). During this test, the particles varied drastically in size so average change in mass and average swelling ratio were needed to draw accurate results. Polymer A had the lowest average swelling ratio with the average initial mass being 0.0186 
Table 2 Results from Mechanical Integrity Testing for Polymers.

\begin{tabular}{|c|c|c|c|c|c|}
\hline \multicolumn{3}{|c|}{ Co-Polymer Molar Concentrations } & \multirow{2}{*}{ Shear } & \multirow{2}{*}{ Elasticity } & \multirow[t]{2}{*}{ Compression } \\
\hline NIPAm & Bis & PVA & & & \\
\hline $90 \%$ & $10 \%$ & $0 \%$ & 0 & 0 & 0 \\
\hline $85 \%$ & $15 \%$ & $0 \%$ & 0 & 0 & 1 \\
\hline $80 \%$ & $20 \%$ & $0 \%$ & 0 & 0 & 1 \\
\hline $75 \%$ & $25 \%$ & $0 \%$ & 1 & 1 & 1 \\
\hline $70 \%$ & $30 \%$ & $0 \%$ & 0 & 0 & 1 \\
\hline $65 \%$ & $35 \%$ & $0 \%$ & 1 & 0 & 1 \\
\hline $60 \%$ & $40 \%$ & $0 \%$ & 0 & 0 & 1 \\
\hline $75 \%$ & $25 \%$ & $30 \%$ & 0 & 1 & 1 \\
\hline $75 \%$ & $25 \%$ & $35 \%$ & 0 & 1 & 1 \\
\hline $75 \%$ & $25 \%$ & $40 \%$ & 1 & 1 & 1 \\
\hline $75 \%$ & $25 \%$ & $42.5 \%$ & 1 & 1 & 1 \\
\hline $75 \%$ & $25 \%$ & $45 \%$ & 0 & 1 & 1 \\
\hline $\begin{array}{c}\text { Ranking } \\
0\end{array}$ & \multirow{2}{*}{\multicolumn{5}{|c|}{$\begin{array}{l}\text { Ranking Description } \\
\text { Polymer unable to withstand applied forces with mechanical integrity } \\
\text { failing before threshold for nanoparticle application is reached } \\
\text { Polymer capable of withstanding applied forces with mechanical integrity } \\
\text { meeting or exceeding threshold for nanoparticle synthesis. }\end{array}$}} \\
\hline 1 & & & & & \\
\hline
\end{tabular}

grams and the average change in mass being 0.0048 grams resulting in a swelling ratio of $28 \%$ (Table 3). This was the lowest swelling ratio with polymer B having a swelling ratio of $32.28 \%$ (Table 4) and polymer $\mathrm{C}$ having an average swelling ratio of $38 \%$ (Table 5). From this test and the averages, we were able to determine that polymer $\mathrm{C}$ had the most desirable absorption properties. While the data and averages indicated a polymer, which performed better than the others, the trends noticed in the data were difficult to discern. The data appeared to lack any significant relationship between the initial mass of the polymer and the change in mass. The only noticeable relationship was that there was a positive relationship between the initial weight and the change in mass, indicating that as a polymer had a higher initial mass, there was a larger change in mass compared to smaller polymers. In the context of the polymer's absorption behaviors, this may indicate that the polymers at the lower weights were oversaturated in the solution and were unable to absorb as much as the larger polymer fragments. Furthermore, the data had a $\mathrm{p}$ value of 0.223473 , indicating no polymer's swelling ratio was able to be definitively identified as more optimal. Ultimately, based on the mechanical testing and swelling testing, a polymer was identified with more desirable characteristics from mechanical integrity to absorption rates.

From the two phases of study conducted, not only was a new material suggested as a replacement to the currently used PTFE, but optimal concentrations for nanoparticle synthesis in drug delivery were determined. PET was determined to have a higher bulk modulus which translated to a sturdier polymer in application. This conclusion was supported by existing research on the mechanical properties of PTFE and PET. According to testing conducted by Phoenix Technologies, the modulus of elasticity for PET which represents an object's resistance to elastic deformation was between 2 and 2.7 GPa. ${ }^{15}$ In comparison, DuPont conducted material analysis on PTFE and found that it had a modulus of elasticity of $66,700 \mathrm{psi}$ which is close to $0.46 \mathrm{GPa} .{ }^{16} \mathrm{~A}$ higher modulus of elasticity indicates a more rigid and composite polymer. Based on past research and the results of the first phase of this study, PET was conclusively determined to be a more rigid polymer, potentially more applicable in venous graft material.
Table 3. Swelling Test Results for Polymer A.

\begin{tabular}{|c|c|c|c|c|c|}
\hline \multirow{2}{*}{$\begin{array}{c}\text { Trial } \\
\text { Number }\end{array}$} & \multicolumn{5}{|c|}{$75 \%$ NIPAM 25\% Bis } \\
\cline { 2 - 6 } & Initial Mass $(\mathrm{g})$ & Final Mass $(\mathrm{g})$ & $\Delta$ Mass $(\mathrm{g})$ & Swelling Ratio & Intact \\
\hline 1 & 0.0071 & 0.0099 & 0.0028 & 0.3944 & 1 \\
\hline 2 & 0.0119 & 0.0131 & 0.0012 & 0.1008 & 1 \\
\hline 3 & 0.0143 & 0.0163 & 0.0020 & 0.1399 & 0 \\
\hline 4 & 0.0178 & 0.0224 & 0.0046 & 0.2584 & 0 \\
\hline 5 & 0.0321 & 0.0382 & 0.0061 & 0.1900 & 0 \\
\hline 6 & 0.0257 & 0.0308 & 0.0051 & 0.1984 & 0 \\
\hline 7 & 0.0280 & 0.0336 & 0.0056 & 0.2000 & 1 \\
\hline 8 & 0.0201 & 0.0260 & 0.0059 & 0.2935 & 0 \\
\hline 9 & 0.0269 & 0.0332 & 0.0063 & 0.2342 & 0 \\
\hline 10 & 0.0132 & 0.0214 & 0.0082 & 0.6212 & 1 \\
\hline 11 & 0.0129 & 0.0176 & 0.0047 & 0.3643 & 1 \\
\hline 12 & 0.0126 & 0.0172 & 0.0046 & 0.3651 & 1 \\
\hline Average & 0.0186 & 0.0233 & 0.0048 & 0.2800 & 0.5 \\
\hline
\end{tabular}

Table 4 Swelling Test Results for Polymer B.

\begin{tabular}{|c|c|c|c|c|c|}
\hline \multirow{2}{*}{$\begin{array}{c}\text { Trial } \\
\text { Number }\end{array}$} & \multicolumn{5}{|c|}{$75 \%$ NIPAM 25\% Bis 40\% PVA } \\
\cline { 2 - 6 } & Initial Mass (g) & Final Mass (g) & $\Delta$ Mass (g) & Swelling Ratio & Intact \\
\hline 1 & 0.0273 & 0.0325 & 0.0052 & 0.1905 & 0 \\
\hline 2 & 0.0314 & 0.0364 & 0.0050 & 0.1592 & 1 \\
\hline 3 & 0.0131 & 0.0183 & 0.0052 & 0.3969 & 0 \\
\hline 4 & 0.0117 & 0.0151 & 0.0034 & 0.2906 & 1 \\
\hline 5 & 0.0427 & 0.0537 & 0.0110 & 0.2576 & 0 \\
\hline 6 & 0.0107 & 0.0148 & 0.0041 & 0.3832 & 1 \\
\hline 7 & 0.0213 & 0.0263 & 0.0050 & 0.2347 & 1 \\
\hline 8 & 0.0129 & 0.0199 & 0.0070 & 0.5426 & 1 \\
\hline 9 & 0.0163 & 0.0203 & 0.0040 & 0.2454 & 1 \\
\hline 10 & 0.0152 & 0.0203 & 0.0051 & 0.3355 & 1 \\
\hline 11 & 0.0180 & 0.0226 & 0.0046 & 0.2556 & 1 \\
\hline 12 & 0.0153 & 0.0242 & 0.0089 & 0.5817 & 0 \\
\hline Average & 0.0197 & 0.0254 & 0.0057 & 0.3228 & 0.6667 \\
\hline
\end{tabular}

Table 5 Swelling Test Results for Polymer C.

\begin{tabular}{|c|c|c|c|c|c|}
\hline \multirow{2}{*}{$\begin{array}{c}\text { Trial } \\
\text { Number }\end{array}$} & \multicolumn{5}{|c|}{$75 \%$ NIPAM $25 \%$ Bis 42.5\% PVA } \\
\cline { 2 - 6 } & Initial Mass (g) & Final Mass (g) & $\Delta$ Mass $(\mathrm{g})$ & Swelling Ratio & Intact \\
\hline 1 & 0.0392 & 0.0461 & 0.0069 & 0.1760 & 0 \\
\hline 2 & 0.0374 & 0.0447 & 0.0073 & 0.1952 & 1 \\
\hline 3 & 0.0168 & 0.0227 & 0.0059 & 0.3512 & 1 \\
\hline 4 & 0.0327 & 0.0396 & 0.0069 & 0.2110 & 0 \\
\hline 5 & 0.0106 & 0.0165 & 0.0059 & 0.5566 & 0 \\
\hline 6 & 0.0092 & 0.0125 & 0.0033 & 0.3587 & 1 \\
\hline 7 & 0.0106 & 0.0153 & 0.0047 & 0.4434 & 0 \\
\hline 8 & 0.0090 & 0.0142 & 0.0052 & 0.5778 & 1 \\
\hline 9 & 0.0096 & 0.0125 & 0.0029 & 0.3021 & 1 \\
\hline 10 & 0.0102 & 0.0152 & 0.0050 & 0.4902 & 1 \\
\hline 11 & 0.0166 & 0.0226 & 0.0060 & 0.3614 & 1 \\
\hline 12 & 0.0097 & 0.0149 & 0.0052 & 0.5361 & 0 \\
\hline Average & 0.0176 & 0.0231 & 0.0054 & 0.3800 & 0.5833 \\
\hline
\end{tabular}

Table ${ }^{6}$. Mean Swelling Ratio for Each Polymer with Standard Deviation.

\begin{tabular}{|c|c|}
\hline & Mean \pm Standard Deviation \\
\hline $75 \%$ NIPAM $25 \%$ & $0.28003 \pm 0.14126$ \\
\hline $75 \%$ NIPAM $25 \% 40 \%$ PVA & $0.32280 \pm 0.13228$ \\
\hline $75 \%$ NIPAM $25 \% 42.5 \%$ PVA & $0.37997 \pm 0.14233$ \\
\hline
\end{tabular}

Poly-NIPAm is a thermo-responsive polymer which has collapsing properties around the temperature range of $37^{\circ} \mathrm{C}$. At that temperature, the polymer shrinks drastically, expelling any solution. Due to this property of Poly-NIPAm, N-isopropylacrylamide was chosen as the base chemical for polymer synthesis and was crosslinked with Bis to form a solid polymer. Poly-vinyl alcohol, a known elastomer, was added to develop a full interpenetrating polymer network (IPN) to not only improve the strength of the polymer but also enhance the swelling properties: Poly-vinyl alcohol was the monomer which was added to the initial polymer synthesized from NI- 
PAm and Bis. The impact of an IPN on the structural integrity of a polymer was demonstrated through mechanical testing of the polymers without PVA and with PVA and the comparison between swelling rates of polymers with a PVA concentration compared to a polymer without it. The polymers with a PVA concentration, on average, had an $85 \%$ higher success rate in passing elastic testing compared to polymers without it and had a $10 \%$ higher success rate in passing shear testing. Furthermore, when subject to swelling tests, polymers with a PVA concentration had on average a $7 \%$ higher swelling rate than polymers without a PVA concentration. A study conducted by researchers from the Institute of Organic and Polymeric Materials, Research and Development Center for Smart Textile Technology, and National Taipei University of Technology characterized the relationship between $\mathrm{N}$-isopropylacrylamide and its thermo-responsive properties. ${ }^{17}$ The researchers found that a semi-IPN structure increased the fracture strain of the polymer by at least $46 \%$ and the swelling and deswelling rates increased significantly for the hydrogels. While the researchers used Itaconamic acid instead of Poly-vinyl alcohol, their research still attests to the possibility of an IPN increasing the mechanical integrity of a polymer and improving swelling/deswelling rates. The effects of Poly-vinyl alcohol and the development of an IPN on the mechanical integrity and absorption rates of synthesized polymers was analyzed and quantified, showing the benefits of developing an interpenetrating polymer network with the monomer PVA in a hydrogel synthesized from NIPAm and Bis.

\section{Conclusion}

The overall takeaways of this experiment lie in the data produced from the Quantum Expresso software Kohn-Sham density functional theory analysis of PET and PTFE and the comparison between the different polymers that were synthesized. When analyzed, the bulk modulus for PET was 105.9 $\mathrm{GPa}$, which was higher than the value that was determined for PTFE by $16.6 \mathrm{GPa}$. With Phase 1 data complete and a mechanical analysis of the polymers finished, it is possible to conclude that $\mathrm{PET}$ is a more rigid polymer compared to PTFE, corroborating the initial hypothesis that PET can replace PTFE in a graft application. The second phase was also completed, and three polymers were determined to be the most viable in terms of rigidity from qualitative strength analysis and one polymer was deemed the most applicable for nanoparticle synthesis based on swelling tests. From polymer variations and qualitative sheer, compression, and elastic testing, the data indicated that polymers created from 75\% NIPAm and 25\% Bis (Polymer A); 75\% NIPAm, 25\% Bis and 40\% PVA (Polymer B); and 75\% NIPAm 25\% Bis and 42.5\% PVA (Polymer C) were the most mechanically sound and held the most promise for future nanoparticle synthesis. With the polymers analyzed and the viable molar concentrations chosen, swelling tests were conducted. Multiple polymers were created from the chosen chemical concentrations. These polymers were then broken into multiple pieces until polymer pieces did not exceed 0.0450 grams. Swelling tests were performed on each piece with a solution which had a $\mathrm{pH}$ of 7.6. Although initial mass was varied purposefully, change in mass and swelling ratio were still representative of which polymers had more desirable swelling capabilities. Data analysis showed that the polymer without any concentration of PVA was the worst performing in terms of average change in mass and had the lowest average number of polymers intact with only $50 \%$ remaining as a single composite piece. The polymers which incorporated PVA had higher changes in mass and more polymers remained intact after the 48-hour period. Polymer B had an average swelling ratio of $32.28 \%$ which was lower than Polymer C whose recorded average swelling ratio was 38\%. Polymer B however had a higher percentage of polymer particles intact after the swelling test in comparison to Polymer C. With this analysis, it can be determined that the polymer concentration with $75 \%$ NIPAm, 25\% Bis, and 42.5\% PVA, Polymer C, may be a more viable polymer concentration for future nanoparticle synthesis and for further study on LCST properties.

\section{Methods}

\section{Method A}

The first phase of the study focused on utilizing Quantum ESPRESSO and Kohn-Sham Density Functional Theory to compare two polymers to determine which one is sturdier and more resistant to deformation. The bulk modulus is a mechanical value which indicates a material's resistance to deformation in gigapascals $(\mathrm{GPa})$. BURAI Software was used to generate input files for the quantum software to run the calculations. From the Materials Project, a ".CIF" file was found for the desired chemicals which describes the crystallographic nature of the compound. BURAI utilizes this file to generate an input file in Fortran code for the quantum program. Certain parameters need to be defined before the input file is ready for computation. The structure of the compound needs to be changed to crystal form and the dimensions of the compound given by "a, b, c" coordinates need to be defined. Furthermore, the parameters for the SCF calculations need to be defined. The max step was set to 200 iterations and the max time allotted for computation was set to 86,000 seconds. Force and Stress parameters were set to "on" and the cutoff parameters for wave function and charge were autogenerated to maintain constants and lower the computational cost. K-Points were also auto-generated. The occupation of the calculation was set to gaussian smearing. With the parameters defined, the input file was run through command prompt which opened the quantum software to run calculations. Each input file corresponded with a singular output file. The output files were searched to find the data point indicating total energy which was denoted by a "!". The total energy was recorded in a table with the corresponding volume. Fifty trials were run per chemical with the "a, b, c" parameters incrementally increased by 0.1 angstrom for each iteration. After fifty trials, the table was then used as an input file for another quantum program which utilized Murnaghan's Equation to compute the bulk modulus of the materials. To run the program, certain parameters needed to be defined: the unit of measurement needed to be defined as 
"Ang", the Bravais lattice structure had to be defined as noncubic, and finally the correct equation needed to be selected from the listed options. The program then generates an output file which gives the bulk modulus of the chemical. This procedure was repeated for each tested compound over a timeline from about November 2019 through January 2020.

\section{Method B}

The second phase focused on the polymerization and synthesis of various polymers and the characterization of the mechanical and absorption properties. $\mathrm{N}$-isopropylacrylamide (NIPAm), N, N'-Methylenebisacrylamide (Bis), Poly-vinyl Alcohol (PVA), Tetramethylethylenediamine (TEMED) and Potassium Persulfate (KPS) were used in the polymerization process. NIPAm was the base polymer with Bis acting as a crosslinker and PVA was later added to create an interpenetrating polymer network. TEMED was the catalyst used to overcome oxygen acting as a retarder for the polymerization process and KPS was the initiator. Polymers were initially synthesized from varying molar concentrations of NIPAm and Bis to create a polymer capable of passing qualitative mechanical testing. For the conduction of the elastic test, the polymer was stretched to its limits to identify the point at which it broke. The shear test was performed by exerting force in directions that were perpendicular to the major axis. The compression test was performed by putting the polymer under an extreme force to see how a large load would impact the structural integrity of the polymer. All of these polymers received a score based on how they performed on the various tests. Polymers were created until one polymer had passed all three mechanical tests and remained a single composite piece. These polymers had varying concentrations of NIPAm and Bis but did not have any amount of PVA. Once a polymer which passed all tests was identified, the concentrations were noted, and new polymers with the same concentrations of NIPAm and Bis were synthesized with varying PVA concentrations. The polymers were once again subjected to qualitative mechanical testing to determine which PVA concentration resulted in the most durable polymers. Once multiple polymers had been identified, they were then broken into pieces within a 1-3-millimeter size range. Next, a solution with a $\mathrm{pH}$ of 7.6 was created by using a sodium phosphate buffer, the monobasic and dibasic components, to mimic the drug which the particles are intended to hold during its application. Once the solution was created, the polymer particles' initial weights were measured, and each particle was placed in a separate well-plate. Twelve particles were tested per polymer. Each particle was then submerged in the solution for 48-hours and after the final weights were recorded, the change in mass was calculated. To determine the approximate swelling rates per polymer type, the change in mass was divide by initial mass and was averaged to determine the mean swelling ratio per polymer. Higher values were indicative of more desirable absorption properties. Trials were run from January 2020 through March 2020.

\section{Acknowledgements}

We thank Dr. Alexis Patanarut (George Mason University) for providing access to the laboratory equipment and offering guidance throughout the duration of this study.

\section{References}

1. Cancer Facts \& Figures (2019). American Cancer Society. American Cancer Society.

2. Marsoner, K., Langeder, R., Csengeri, D., Sodeck, G., Mischinger, H. J., and Kornprat, P. (2016, August) Portal vein resection in advanced pancreatic adenocarcinoma: is it worth the risk? Wiener klinische Wochenschrift. Springer Vienna.

3. Alemi, F., Rocha, F. G., Helton, W. S., Biehl, T., and Alseidi, A. (2016, October) Classification and techniques of en bloc venous reconstruction for pancreaticoduodenectomy. HPB : the official journal of the International Hepato Pancreato Biliary Association. Elsevier.

4. Kim, S. M., Min, S.-K., Park, D., Min, S.-I., Jang, J.-Y., Kim, S.-W., Ha, J., and Kim, S. J. (2013, June) Reconstruction of portal vein and superior mesenteric vein after extensive resection for pancreatic cancer. Journal of the Korean Surgical Society. The Korean Surgical Society.

5. Kleive, D., Berstad, A. E., Verbeke, C. S., Haugvik, S. P., Gladhaug, I. P., Line, P.-D., and Labori, K. J. (2016, July) Cold-stored cadaveric venous allograft for superior mesenteric/portal vein reconstruction during pancreatic surgery. HPB : the official journal of the International Hepato Pancreato Biliary Association. Elsevier.

6. Dua, M. M., Tran, T. B., Klausner, J., Hwa, K. J., Poultsides, G. A., Norton, J. A., and Visser, B. C. (2015, December 16) Pancreatectomy with vein reconstruction: technique matters. HPB. Elsevier.

7. Using DFT to Predict the Equilibrium Lattice Parameter and Bulk Modulus of Crystalline Materials. Materials Science and Engineering at Illinois. University of Illinois.

8. Choudhary, K., Cheon, G., Reed, E., and Tavazza, F. (2018) Elastic properties of bulk and low-dimensional materials using Van der Waals density functional. Physical review. B. U.S. National Library of Medicine.

9. Skylaris, C.-K. The self-consistent field procedure for Kohn-Sham DFT calculations. CHEM6085: Density Functional Theory. University of Southampton.

10.Lanzalaco, S., and Armelin, E. (2017, October 4) Poly (N-isopropylacrylamide) and Copolymers: A Review on Recent Progresses in Biomedical Applications. Gels (Basel, Switzerland). MDPI.

11.Hu, X., Tong, Z., and Lyon, L. A. (2011, April 5) Control of poly ( $\mathrm{N}$-isopropylacrylamide) microgel network structure by precipitation polymerization near the lower critical solution temperature. Langmuir : the ACS journal of surfaces and colloids. U.S. National Library of Medicine.

12. 2001, January 5) SDS Page Gel Electrophoresis. SDS-PAGE. Georgia Tech.

13.Nagase, K., Yamato, M., Kanazawa, H., and Okano, T. (2017, October 27) Poly(N-isopropylacrylamide)-based thermoresponsive surfaces provide new types of biomedical applications. Biomaterials. Elsevier.

14.Peralta, M. E., Jadhav, S. A., Magnacca, G., Scalarone, D., Mártire, D. O., Parolo, M. E., and Carlos, L. (2019, February 26) Synthesis and in vitro testing of thermoresponsive polymer-grafted core-shell magnetic mesoporous silica nanoparticles for efficient controlled and targeted drug delivery. Journal of Colloid and Interface Science. Academic Press.

15.(2008) Polyethylene terephthalate Key Properties. PET Properties. Phoenix Technologies.

16. Teflon PTFE. Properties Handbook. Dupont.

17. Rwei, S.-P., Tuan, H. N. A., Chiang, W.-Y., and Way, T.-F. (2018, April 28) Synthesis and Characterization of $\mathrm{pH}$ and Thermo Dual-Responsive Hydrogels with a Semi-IPN Structure Based on N-Isopropylacrylamide and Itaconamic Acid. Materials (Basel, Switzerland). MDPI.

\section{Authors}

Anish Pothireddy is a high school senior who attends the Governor's School at George Mason University. He is currently studying university physics with the intention of achieving 
a double-major in computer science and biology with a concentration in machine learning. He has completed multiple research projects including one for NASA.

Samyak Thapa is a high school senior who attends the Governor's School at Innovation Park and Colgan High School. $\mathrm{He}$ is currently pursuing an advanced diploma with a focus on physics at the Governor's School. He plans on majoring in computer engineering in college with a minor in finance.

Vishnu Lakshmanan is a high school senior who attends the Governor's School at Innovation Park pursuing an advanced studies diploma. He is currently enrolled in University Physics and plans on graduating with a computer science major coupled with an economics minor.

\section{Appendix}

Appendix Table 1: Polyethylene Terephthalate Data Table.

\begin{tabular}{|c|c|c|c|c|c|}
\hline $\begin{array}{c}\text { Trial } \\
\text { Number }\end{array}$ & $\begin{array}{c}\text { A Parameter } \\
\text { (Ang) }\end{array}$ & \begin{tabular}{|c|}
$\begin{array}{c}\text { B Parameter } \\
\text { (Ang) }\end{array}$ \\
\end{tabular} & $\begin{array}{c}\text { C Parameter } \\
\text { (Ang) }\end{array}$ & $\begin{array}{c}\text { Volume } \\
\text { (Ang^3) }\end{array}$ & $\begin{array}{c}\text { Total Energy } \\
\text { (Ry) }\end{array}$ \\
\hline 1 & 2.07952 & 3.50216 & \begin{tabular}{|l|}
6.61977 \\
\end{tabular} & 48.21054 & -215.79353 \\
\hline 2 & 2.17952 & 3.60216 & 6.71977 & 52.75678 & -220.39223 \\
\hline 3 & 2.27952 & 3.70216 & 6.81977 & 57.55305 & -224.43422 \\
\hline 4 & 2.37952 & 3.80216 & 6.91977 & 62.60534 & -227.99551 \\
\hline 5 & 2.47952 & 3.90216 & 7.01977 & 67.91967 & -231.13005 \\
\hline 6 & 2.57952 & 4.00216 & 7.11977 & 73.50203 & -233.89658 \\
\hline 7 & 2.67952 & 4.10216 & 7.21977 & 79.35841 & -236.32952 \\
\hline 8 & 2.77952 & 4.20216 & 7.31977 & 85.49482 & -238.46832 \\
\hline 9 & 2.87952 & 4.30216 & 7.41977 & 91.91727 & -240.34570 \\
\hline 10 & 2.97952 & 4.40216 & 7.51977 & 98.63174 & -241.99388 \\
\hline 11 & 3.07952 & 4.50216 & 7.61977 & 105.64424 & -243.43425 \\
\hline 12 & 3.17952 & 4.60216 & 7.71977 & 112.96077 & -244.68476 \\
\hline 13 & 3.27952 & 4.70216 & 7.81977 & 120.58733 & -245.77257 \\
\hline 14 & 3.37952 & 4.80216 & 7.91977 & \begin{tabular}{|l|}
128.52991 \\
\end{tabular} & -246.71042 \\
\hline 15 & 3.47952 & 4.90216 & 8.01977 & \begin{tabular}{|l|}
136.79453 \\
\end{tabular} & -247.51682 \\
\hline 16 & 3.57952 & 5.00216 & 8.11977 & 145.38718 & -248.20552 \\
\hline 17 & 3.67952 & 5.10216 & 8.21977 & 154.31385 & -248.78838 \\
\hline 18 & 3.77952 & 5.20216 & 8.31977 & 163.58055 & -249.27420 \\
\hline 19 & 3.87952 & 5.30216 & 8.41977 & 173.19329 & -249.67922 \\
\hline 20 & 3.97952 & 5.40216 & 8.51977 & 183.15805 & -250.00430 \\
\hline 21 & 4.07952 & 5.50216 & 8.61977 & \begin{tabular}{|l|}
193.48084 \\
\end{tabular} & -250.26495 \\
\hline 22 & 4.17952 & 5.60216 & 8.71977 & \begin{tabular}{|l|}
204.16766 \\
\end{tabular} & -250.46238 \\
\hline 23 & 4.27952 & 5.70216 & 8.81977 & \begin{tabular}{|l|}
215.22451 \\
\end{tabular} & -250.60966 \\
\hline 24 & 4.37952 & 5.80216 & 8.91977 & 226.65738 & -250.70726 \\
\hline 25 & 4.47952 & 5.90216 & 9.01977 & \begin{tabular}{|l|}
238.47229 \\
\end{tabular} & -250.76240 \\
\hline 26 & 4.57952 & 6.00216 & 9.11977 & 250.67523 & -250.77873 \\
\hline 27 & 4.67952 & 6.10216 & 9.21977 & \begin{tabular}{|l|}
263.27219 \\
\end{tabular} & -250.76075 \\
\hline 28 & 4.77952 & 6.20216 & 9.31977 & \begin{tabular}{|l|}
276.26918 \\
\end{tabular} & -250.71481 \\
\hline 29 & 4.87952 & 6.30216 & 9.41977 & 289.67221 & -250.64267 \\
\hline 30 & 4.97952 & 6.40216 & 9.51977 & 303.48726 & -250.54741 \\
\hline 31 & 5.07952 & 6.50216 & 9.61977 & \begin{tabular}{|l|}
317.72034 \\
\end{tabular} & -250.43258 \\
\hline 32 & 5.17952 & 6.60216 & 9.71977 & 332.37745 & -250.30032 \\
\hline 33 & 5.27952 & 6.70216 & 9.81977 & \begin{tabular}{|l|}
347.46459 \\
\end{tabular} & -250.15244 \\
\hline 34 & 5.37952 & 6.80216 & 9.91977 & 362.98775 & -249.99306 \\
\hline 35 & 5.47952 & 6.90216 & 10.01977 & \begin{tabular}{|l|}
378.95295 \\
\end{tabular} & -249.82281 \\
\hline 36 & 5.57952 & 7.00216 & 10.11977 & 395.36617 & -249.64426 \\
\hline 37 & 5.67952 & 7.10216 & 10.21977 & 412.23343 & -249.45775 \\
\hline 38 & 5.77952 & 7.20216 & 10.31977 & \begin{tabular}{|l|}
429.56071 \\
\end{tabular} & -249.26670 \\
\hline 39 & 5.87952 & 7.30216 & 10.41977 & \begin{tabular}{|l|}
447.35403 \\
\end{tabular} & -249.06949 \\
\hline 40 & 5.97952 & 7.40216 & 10.51977 & 465.61937 & -248.86996 \\
\hline 41 & 6.07952 & 7.50216 & 10.61977 & \begin{tabular}{|l|l|}
484.36274 \\
\end{tabular} & -248.66858 \\
\hline 42 & 6.17952 & 7.60216 & 10.71977 & 503.59014 & -248.46513 \\
\hline 43 & 6.27952 & 7.70216 & 10.81977 & \begin{tabular}{|l|}
523.30757 \\
\end{tabular} & -248.26278 \\
\hline 44 & 6.37952 & 7.80216 & 10.91977 & \begin{tabular}{|l}
543.52102 \\
\end{tabular} & -248.05979 \\
\hline 45 & 6.47952 & 7.90216 & 11.01977 & 564.23651 & -247.85751 \\
\hline 46 & 6.57952 & 8.00216 & 11.11977 & 585.46002 & -247.65652 \\
\hline 47 & 6.67952 & 8.10216 & 11.21977 & 607.19757 & -247.45739 \\
\hline 48 & 6.77952 & 8.20216 & 11.31977 & 629.45514 & -247.26177 \\
\hline 49 & 6.87952 & 8.30216 & 11.41977 & 652.23874 & -247.06832 \\
\hline 50 & 6.97952 & 8.40216 & 11.51977 & 675.55438 & -246.87875 \\
\hline
\end{tabular}

Appendix Table 2: Polytetrafluoroethylene Data Table.

\begin{tabular}{|c|c|c|c|c|c|}
\hline $\begin{array}{c}\text { Trial } \\
\text { Number }\end{array}$ & $\begin{array}{c}\text { Dimension A } \\
\text { (Ang) }\end{array}$ & \begin{tabular}{|c|} 
Dimension B \\
(Ang)
\end{tabular} & \begin{tabular}{|c|} 
Dimension C \\
(Ang)
\end{tabular} & $\begin{array}{l}\text { Volume } \\
\text { (Ang^3) }\end{array}$ & \begin{tabular}{|c} 
Total Energy \\
(Ry)
\end{tabular} \\
\hline 1 & 3.70699 & 3.87373 & 4.22379 & 60.65311 & \begin{tabular}{|l}
-610.81039 \\
\end{tabular} \\
\hline 2 & 3.80699 & 3.97373 & 4.32379 & 65.41008 & \begin{tabular}{|l|}
-616.00436 \\
\end{tabular} \\
\hline 3 & 3.90699 & 4.07373 & 4.42379 & 70.40914 & $\begin{array}{l}-620.57813 \\
\end{array}$ \\
\hline 4 & 4.00699 & 4.17373 & 4.52379 & 75.65629 & \begin{tabular}{|l|}
-624.60439 \\
\end{tabular} \\
\hline 5 & 4.10699 & 4.27373 & 4.62379 & 81.15753 & \begin{tabular}{|l}
-628.14814 \\
\end{tabular} \\
\hline 6 & 4.20699 & 4.37373 & 4.72379 & 86.91886 & \begin{tabular}{|l}
-631.26586 \\
\end{tabular} \\
\hline 7 & 4.30699 & 4.47373 & 4.82379 & 92.94628 & $\begin{array}{l}-634.00688 \\
\end{array}$ \\
\hline 8 & 4.40699 & 4.57373 & 4.92379 & \begin{tabular}{|l|}
99.24579 \\
\end{tabular} & \begin{tabular}{|l}
-636.41529 \\
\end{tabular} \\
\hline 9 & 4.50699 & 4.67373 & 5.02379 & 105.82340 & -638.52956 \\
\hline 10 & 4.60699 & 4.77373 & 5.12379 & 112.68509 & -640.38384 \\
\hline 11 & 4.70699 & 4.87373 & 5.22379 & 1119.83687 & -642.00821 \\
\hline 12 & 4.80699 & 4.97373 & 5.32379 & 127.28474 & $\begin{array}{l}-643.42902 \\
\end{array}$ \\
\hline 13 & 4.90699 & 5.07373 & 5.42379 & 135.03470 & \begin{tabular}{|l|}
-644.66996 \\
\end{tabular} \\
\hline 14 & 5.00699 & 5.17373 & 5.52379 & 143.09275 & $\begin{array}{l}-645.75146 \\
\end{array}$ \\
\hline 15 & 5.10699 & 5.27373 & 5.62379 & 151.46490 & $\begin{array}{l}-646.69180 \\
\end{array}$ \\
\hline 16 & 5.20699 & 5.37373 & 5.72379 & 160.15713 & -647.50694 \\
\hline 17 & 5.30699 & 5.47373 & 5.82379 & 169.17545 & \begin{tabular}{|l}
-648.21110 \\
\end{tabular} \\
\hline 18 & 5.40699 & 5.57373 & 5.92379 & \begin{tabular}{|l|}
178.52587 \\
\end{tabular} & -648.81671 \\
\hline 19 & 5.50699 & 5.67373 & 6.02379 & \begin{tabular}{|l}
188.21437 \\
\end{tabular} & \begin{tabular}{|c|}
-649.3348 \\
\end{tabular} \\
\hline 20 & 5.60699 & 5.77373 & 6.12379 & \begin{tabular}{|l|}
198.24696 \\
\end{tabular} & \begin{tabular}{|l}
-649.775466 \\
\end{tabular} \\
\hline 21 & 5.70699 & 5.87373 & 6.22379 & 208.62965 & $\begin{array}{l}-650.14710 \\
\end{array}$ \\
\hline 22 & 5.80699 & 5.97373 & 6.32379 & 219.36842 & \begin{tabular}{|l}
-650.45760 \\
\end{tabular} \\
\hline 23 & 5.90699 & 6.07373 & 6.42379 & 230.46928 & \begin{tabular}{|l}
-650.71386 \\
\end{tabular} \\
\hline 24 & 6.00699 & 6.17373 & 6.52379 & 241.93824 & \begin{tabular}{|l}
-650.92204 \\
\end{tabular} \\
\hline 25 & 6.10699 & 6.27373 & 6.62379 & 253.78128 & $\begin{array}{l}-651.08762 \\
\end{array}$ \\
\hline 26 & 6.20699 & 6.37373 & 6.72379 & \begin{tabular}{|l|}
266.00442 \\
\end{tabular} & $\begin{array}{l}-651.21546 \\
\end{array}$ \\
\hline 27 & 6.30699 & 6.47373 & 6.82379 & 278.61364 & \begin{tabular}{|l}
-651.30990 \\
\end{tabular} \\
\hline 28 & 6.40699 & 6.57373 & 6.92379 & \begin{tabular}{|l|}
291.61496 \\
\end{tabular} & $\begin{array}{l}-651.37483 \\
\end{array}$ \\
\hline 29 & 6.50699 & 6.67373 & 7.02379 & 305.01436 & \begin{tabular}{|l}
-651.41373 \\
\end{tabular} \\
\hline 30 & 6.60699 & 6.77373 & 7.12379 & 318.81786 & \begin{tabular}{|l}
-651.42968 \\
\end{tabular} \\
\hline 31 & 6.70699 & 6.87373 & 7.22379 & \begin{tabular}{|l|}
333.03144 \\
\end{tabular} & -651.42545 \\
\hline 32 & 6.80699 & 6.97373 & 7.32379 & 347.66112 & \begin{tabular}{|l}
-651.40356 \\
\end{tabular} \\
\hline 33 & 6.90699 & 7.07373 & 7.42379 & \begin{tabular}{|l|}
362.71289 \\
\end{tabular} & $\begin{array}{l}-651.36623 \\
\end{array}$ \\
\hline 34 & 7.00699 & 7.17373 & 7.52379 & \begin{tabular}{|l|}
378.19274 \\
\end{tabular} & \begin{tabular}{|l|}
-651.31550 \\
\end{tabular} \\
\hline 35 & 7.10699 & 7.27373 & 7.62379 & 394.10669 & \begin{tabular}{|l}
-651.25317 \\
\end{tabular} \\
\hline 36 & 7.20699 & 7.37373 & 7.72379 & \begin{tabular}{|l|l|}
410.46073 \\
\end{tabular} & $\begin{array}{l}-651.18092 \\
\end{array}$ \\
\hline 37 & 7.30699 & 7.47373 & 7.82379 & 427.26085 & \begin{tabular}{|l|}
-651.10021 \\
\end{tabular} \\
\hline 38 & 7.40699 & 7.57373 & 7.92379 & \begin{tabular}{|l|}
444.51307 \\
\end{tabular} & $\begin{array}{l}-651.01241 \\
\end{array}$ \\
\hline 39 & 7.50699 & 7.67373 & 8.02379 & 462.22338 & $\begin{array}{l}-650.91874 \\
\end{array}$ \\
\hline 40 & 7.60699 & 7.77373 & 8.12379 & 480.39777 & \begin{tabular}{|l}
-650.82029 \\
\end{tabular} \\
\hline 41 & 7.70699 & 7.87373 & 8.22379 & 499.04226 & \begin{tabular}{|l}
-650.71808 \\
\end{tabular} \\
\hline 42 & 7.80699 & 7.97373 & 8.32379 & \begin{tabular}{|l|}
518.16284 \\
\end{tabular} & \begin{tabular}{|l}
-650.61305 \\
\end{tabular} \\
\hline 43 & 7.90699 & 8.07373 & 8.42379 & \begin{tabular}{|l|}
537.76551 \\
\end{tabular} & $\begin{array}{l}-650.50601 \\
\end{array}$ \\
\hline 44 & 8.00699 & 8.17373 & 8.52379 & 557.85627 & $\begin{array}{l}-650.39776 \\
\end{array}$ \\
\hline 45 & 8.10699 & 8.27373 & 8.62379 & 578.44111 & \begin{tabular}{|l|}
-650.288991 \\
\end{tabular} \\
\hline 46 & 8.20699 & 8.37373 & 8.72379 & 599.52605 & \begin{tabular}{|l}
-650.18006 \\
\end{tabular} \\
\hline 47 & 8.30699 & 8.47373 & 8.82379 & \begin{tabular}{|l|}
621.11708 \\
\end{tabular} & $\begin{array}{l}-650.07176 \\
\end{array}$ \\
\hline 48 & 8.40699 & 8.57373 & 8.92379 & \begin{tabular}{|l|}
643.22020 \\
\end{tabular} & -649.96453 \\
\hline 49 & 8.50699 & 8.67373 & 9.02379 & \begin{tabular}{|l|}
665.84141 \\
\end{tabular} & -649.85909 \\
\hline 50 & 8.60699 & 8.77373 & 9.12379 & \begin{tabular}{|l|}
688.98671 \\
\end{tabular} & -649.75618 \\
\hline
\end{tabular}

